\title{
Rezension: Stefan Schlegel, Der Entscheid über Migration als Verfügungsrecht
}

Philip C. Hanke *

Ein Recht ist ein Gut. Und als solches hat es einen Wert bzw. einen Preis. Selbiges, so die zentrale Annahme des vorliegenden Buches, gilt auch für das Recht, über Migration zu entscheiden. Wer jedoch soll dieses Recht bekommen? Die Zielstaaten, die Herkunftsstaaten oder die Migrierenden? Was auf den ersten Blick wie eine recht unübliche Frage wirkt - sind es doch traditionell immer die Zielstaaten, die in ihrer Souveränität über die Zulassung von Migration entscheiden -, ist bei näherem Betrachten nicht so offenkundig. Ist es denn überhaupt sinnvoll, dass eben diese Zielstaaten dieses Recht quasi automatisch zugeteilt bekommen?

Zitiervorschlag: Philip C. Hanke, Rezension: Stefan Schlegel, Der Entscheid über Migration als Verfügungsrecht, in: sui-generis 2017, S. 57

URL: $\quad$ sui-generis.ch/33

DOI: $\quad$ https://doi.org/10.21257/sg.33

* Dr. Philip C. Hanke, LL.M. (EMLE), ist PostDoc an der Universität Bern und im nationalen Forschungsschwerpunkt «On the Move» des schweizerischen Nationalfonds tätig. 
Dieser sehr grundsätzlichen Frage, nämlich wer denn eigentlich über Migration entscheiden dürfen soll, geht Schlegel in seiner am Institut für öffentliches Recht der Universität Bern entstandenen und nun bei Mohr Siebeck erschienenen Dissertation nach. Es geht ihm dabei nicht direkt um die dahintersteckenden politischen Prozesse, sondern um sehr prinzipielle, normative Fragen. Um diese zu beantworten, braucht es eine ausgeklügelte Methode - und diese ist in dem Fall die ökonomische Analyse des Rechts, also das Anwenden von ökonomischer Theorie auf Fragen der optimalen Entscheidungsregeln. Schlegels Analyse findet primär im Schweizer Migrationsrecht statt, wenngleich ein paar Bezüge zu Entwicklungen im Ausland, insbesondere in Europa (v.a. zum Personenfreizügigkeitsabkommen zwischen der Schweiz und der EU), hergestellt werden. Auch grenzt er die Arbeit in erster Linie auf die Migration von unselbständig Erwerbstätigen ein (wobei die Gedanken sich problemlos auch auf andere Formen von Migration erweitern lassen).

Da die Arbeit eine theoretisch-konzeptionelle auf dem Gebiet der ökonomischen Analyse des Rechts ist, nimmt sich Schlegel eingangs den Raum, die Leserin bzw. den Leser ausführlich in die Methode einzuführen. Das erste Kapitel macht es sich dabei explizit zur Aufgabe, die grundlegenden Begriffe und Literaturstränge zu vermitteln, sodass auch LeserInnen ohne Vorwissen den Ausführungen und Überlegungen im Rest der Arbeit gut folgen können. Danach macht er sich intensiv Gedanken, wie man die ökonomische Theorie der Verfügungsrechte auf das Migrationsrecht anwenden kann. Dabei ist nicht nur wichtig, wem das Recht zuerst zugeteilt wird, sondern auch, wie es dann in einem nächsten Schritt übertragen werden kann. Zentral ist hierbei, dass es sich beim Migrationsrecht in Wirklichkeit um ein Rechtebündel handelt: Schlegel konzentriert sich bewusst auf den Zugang zum Arbeitsmarkt, allerdings gehören Fragen wie die des Familiennachzugs, des Zugangs zum Sozialstaat oder des Bürgerrechts auch zu diesem Bündel an Rechten.

3 In der gängigen Praxis heutzutage liegt das Verfügungsrecht beim Zielstaat, der entsprechend einem «Abwehrregel» genanntem Prinzip (property rule in der englischsprachigen Literatur, auf welcher diese Theorie beruht) Zuwanderung prinzipiell verbieten kann, dieses Recht aber auch, so er denn will, an andere übertragen kann. Das privatrechtliche Äquivalent wäre beispielsweise eine Regel, die es HausbesitzerInnen erlaubt, mittels einer gerichtlichen Verfügung gegen eine benachbarte Fabrik, die Verschmutzung verursacht, vorzugehen. Stattdessen, so Schlegel, könnte die Übertragungsregel auch anders aussehen und viel mehr in Form einer Entschädigungsregel ausgestaltet sein (liability rule). So wie ein Gericht befinden könnte, dass es die Verschmutzung nicht unterbindet (z.B. weil die Aktivität der Fabrik aus einer gesamtgesellschaftlichen Perspektive gewünscht wird), aber den HausbesitzerInnen Entschädigungszahlungen zuspricht. Angewandt auf das Migrationsrecht hiesse das, das Verfügungsrecht über Migration wäre den Zielstaaten zugesprochen, Migrierende könnten sich aber darüber hinwegsetzen, indem sie dem Zielstaat eine Entschädigung zahlen, beispielsweise in Form einer Sondersteuer. Schlegel beschreibt 
hierbei mehrere mögliche Szenarien und diskutiert im Detail die unterschiedlichen Möglichkeiten, welche Vor- und Nachteile für die jeweiligen Kombinationen aus primärer Zuteilung und Übertragungsregel mit sich bringen. Schliesslich wendet Schlegel diese Taxonomie auf die Spezifika des Schweizer Migrationsrechts an. Das zentrale Kriterium in der Bewertung ist die Effizienz. So wie es gesamtgesellschaftlich gesehen nicht immer effizient wäre, wenn NachbarInnen umweltverschmutzende Fabriken zum Stillstand zwingen könnten, können falsche Zuteilungen von Verfügungsrechten und Übertragungsregeln im Migrationsrecht zu schlechten Ergebnissen führen.

Wichtig ist, die gegenseitigen Effekte zu sehen: Migration hat Auswirkungen auf das Zielland, aber die Verhinderung von Migration hat Auswirkungen auf die Personen, die migrieren wollen. Die Verfügungsrechte sollten also so verteilt sein, dass der Gesamtschaden minimiert wird, und sie sollten leicht übertragbar sein. Das Verfügungsrecht über Migration soll dementsprechend denjenigen zugesprochen werden, die es am meisten schätzen. Das gegenwärtige Schweizer Migrationsrecht nimmt nicht vollständig Rücksicht auf die negativen Externalitäten (also Effekte auf Aussenstehende), die es verursacht. Die Schlussfolgerung, ohne hier zu viel verraten zu wollen, ist, dass Ressourcen ineffizient verwendet werden - Schlegel untermauert dies gut mit dem Beispiel eines Asylsuchenden, der seit vielen Jahren in der Schweiz wohnt, aber keiner Arbeit nachgehen durfte und deshalb auf öffentliche Hilfe angewiesen war, obwohl er in dieser langen Zeit sich gut hätte etablieren können.
5 Die Arbeit, mit ihrer klar definierten geographischen Eingrenzung auf die Schweiz, zeigt hierbei auch gut, wie schwierig es ist, die optimale Entscheidungsebene für eine Effizienzanalyse zu finden. Was für die lokale Ebene stimmen mag, ist für die nationale oder supranationale Ebene nicht notwendigerweise richtig. Ein wichtiger grundlegender Unterschied zwischen dem Migrationsrecht und anderen Rechtsgebieten ist die Frage, wer die Entscheidung über die Zuteilung von Verfügungsrechten trifft. Werden solche Entscheidungen normalerweise hinter einem vermeintlichen Schleier der Unwissenheit getroffen - es sind z.B. grundsätzlich nicht die Opfer von Autounfällen, die das Haftpflichtrecht gestalten -, so ist das im Migrationsrecht anders. Die politische Entscheidung über Letzteres wird nämlich traditionell den StaatsbürgerInnen zugesprochen, also, vereinfacht gesagt, jenen, die nicht migrieren (bzw. bei denen die Migration lange her ist). Eine seltene Ausnahme hierbei stellt die EU dar: Hier wurde dieses normalerweise national verortete Verfügungsrecht in ein europäisches Grundrecht umgewandelt, welches von den europäischen Institutionen hochgehalten und notfalls durchgesetzt wird. Gleichzeitig kämpft die aktuelle EuGH-Rechtsprechung auch mit dem Problem, das Rechtebündel genau zu definieren, beispielsweise inwieweit das Aufenthaltsrecht mit dem $\mathrm{Zu}-$ gang zu sozialstaatlichen Leistungen verknüpft ist. ${ }^{1}$ Auf diese Komponente der Mehrstufigkeit des Migrationsrechts, d.h. die Tatsache, dass dieses Rechtsgebiet nicht nur auf der nationalen, sondern auch auf kommunalen, regionalen oder supranationalen Ebenen angesiedelt sein

1 Urteil des Gerichtshofs C-333/13 vom 11. November 2014 (Dano gegen Jobcenter Leipzig). 
kann, wird in Schlegels Arbeit nur kurz eingegangen. Letztlich wäre dem Aspekt, dass Verfügungsrechte durch Instrumente zur Selbstbindung (sogenannte commitment devices), wie etwa die Europäische Union, mehr oder weniger permanent übertragen werden können, durchaus mehr Raum beizumessen. Souveräne Staaten können gewisse Kompetenzen an eine höhergeordnete Ebene abtreten und somit ihren Handlungsspielraum freiwillig einschränken (Beispiele hierfür wären etwa das europäische Beihilfenrecht oder eben das Grundrecht auf freien Personenverkehr). Die Entwicklungen rund um den «Brexit» zeigen denn auch, wie teuer es werden kann, dieses Verfügungsrecht wieder zurückerlangen zu wollen.

Schlegels Argument, dass das Recht über Migration einen Vermögenswert darstellt und ressourcenoptimierend (also: effizient) vergeben werden muss, hat eine Schwachstelle, die nicht Schlegels Mühen geschuldet ist, sondern der Methode: letztlich sind viele Fragen nur sehr schwer in aller Vollständigkeit konzeptionell zu beantworten und verlangen eine Menge an Empirie. Sind die Auswirkungen von Migration positiv oder negativ? Wer empfindet das wie? Wie werden das Recht auf Migration bzw. ein Abwehrrecht dagegen tatsächlich bewertet? Wie ist das jetzt tatsächlich mit der angeblichen «Ausländerkriminalität»? Wenn das Recht ein Gut ist, hat es einen Preis. Und als solches sollte es denjenigen zukommen, die es am meisten schätzen (in der Sprache der Volkswirtschaftslehre: denjenigen, die am meisten dafür bereit sind zu zahlen). Was dieser Preis dann aber schlussendlich ist, kann die Rechtswissenschaft nicht beantworten. In diesem Sinne stellt die Arbeit eine Einla- dung dar, nun den nächsten Schritt zu tätigen, nämlich die Systematik zu verwenden, um dann mit der notwendigen, methodologisch allerdings auch sehr schwierigen Empirie zu klaren Antworten zu kommen.

7 Die Feststellung, dass das Recht ein privates Gut und die Zuteilung von grosser Wichtigkeit ist, ist nicht neu. Dennoch ist das Anwenden der Theorie der Verfügungsrechte auf das Migrationsrecht innovativ, weil diese Theorie in den über 40 Jahren ihrer Existenz noch nie - und schon gar nicht in der Systematik (knapp 400 Seiten) - im Kontext des Migrationsrechts diskutiert wurde. Viele von den Ergebnissen hätte man vielleicht auch ohne den Umweg über die ökonomische Theorie der Verfügungsrechte haben können. Dennoch bietet Schlegel viele gute, neue und wohl durchdachte Denkanstösse, welche die Diskussion voranbringen können. Der Weg ist hier zugleich auch das Ziel, womit die absolut lesenswerte und gut geschriebene Arbeit auch ganz nah an der Realität vieler MigrantInnen ist.

«Der Entscheid über Migration als Verfügungsrecht», Stefan Schlegel, Mohr Siebeck Verlag, 2017, ISBN 978-3-16154872-7 